\title{
Multislice electron ptychography enables lattice vibration-limited resolution and linear phase-contrast imaging in thick samples
}

Zhen Chen ${ }^{1}$, Yi Jiang ${ }^{2}$, Yu-Tsun Shao ${ }^{3}$, Megan Holtz ${ }^{4}$, Michal Odstrcil ${ }^{5}$, Manuel Guizar-Sicairos ${ }^{5}$, Isabelle Hanke $^{6}$, Steffen Ganschow ${ }^{6}$, Darrell Schlom ${ }^{7}$ and David Muller ${ }^{8}$

${ }^{1}$ School of Applied and Engineering Physics, Cornell University, Ithaca, NY, USA, New York, United States,

${ }^{2}$ Advanced Photon Source, Argonne National Laboratory, Lemont, IL 60439, USA, United States, ${ }^{3}$ School of Applied and Engineering Physics, Cornell University, Ithaca, NY, USA, Ithac, New York, United States, ${ }^{4}$ Department of Materials Science and Engineering, Cornell University, Ithaca, NY, USA, United States, ${ }^{5}$ Paul Scherrer Institut, 5232 Villigen PSI, Switzerland, Switzerland, ${ }^{6}$ Leibniz-Institut für Kristallzüchtung, MaxBorn-Str. 2, 12489 Berlin, Germany, Berlin, Germany, ${ }^{7}$ Department of Materials Science and Engineering, Cornell University, Ithaca, NY, USA, Ithaca, New York, United States, ${ }^{8}$ School of Applied and Engineering Physics, Cornell University, Ithaca, NY, USA, Ithaca, New York, United States

The ultimate limit to spatial resolution in an electron microscope is set by the thermal vibrations of the atoms themselves, which are on the order of $10-20 \mathrm{pm}$ at room temperature [1]. Using multislice electron ptychography, we are surprisingly close to this limit. Although conventional annular dark-field imaging has reached spatial resolutions better than $50 \mathrm{pm}$ [2,3], such performance can only be realized in thin samples, usually only a few nanometers thick and after the correction of high-order aberrations. In thicker samples, strong multiple scattering changes the probe shape and causes dechannelling effects between neighboring atomic columns which reduces the interpretable resolution [4]. Multiple scattering also leads to a complicated image contrast that is nonlinearly or even nonmonotonically dependent on the sample thickness, especially for phase-contrast imaging methods.

Ptychography uses scanning diffraction and 4D-STEM datasets to iteratively reconstruct the electrostatic potential and has reached a resolution of $39 \mathrm{pm}$ in thin 2D materials [5]. However, conventional ptychography (single-slice) assumes that the exit-surface wave function can be expressed as a multiplication of the incident probe and a single complex transmission function whose phase represents the sample projected potential. This approximation usually fails for samples thicker than a few nanometers in the presence of strong multiple scattering. For thick samples, attempts at phase retrieval from both multi-slice electron ptychography [6-8] and Bloch wave based scattering matrix [9] approaches have been reported. However, to date, none of the experimental demonstrations have been widely adopted due to limited resolution or image quality.

Here, we demonstrate a robust experimental realization of inversion of the multiple scattering using a regularized implementation of multislice electron ptychography [10]. This approach provides ultra-highresolution reconstructions for samples thicker than tens of nanometers (Figure 1(b)). More importantly, the contrast maintains a linear dependence on thickness over a wide thickness range (Figure 1(c)). In contrast, conventional phase-contrast imaging techniques such as single-slice ptychography or high-resolution TEM (HRTEM) have much worse resolution and provide image contrast not directly interpretable for samples thicker than a few nanometers (Figure 1(b) and (d)). The linear phase-contrast can also greatly widen the applicable sample thickness and resolution for phase-contrast tomography. Furthermore, three-dimensional structural information such as locations of single dopants can also be obtained, since multislice ptychography retrieves the potential of the sample at different depth positions separately.

The potential reconstructed from multislice ptychography from an experimental dataset acquired from a 21nm-thick $\mathrm{PrScO}_{3}$ sample (Figure 2(a)), similar to that from a simulated dataset (Figure 2(d)), shows only a slightly additional blurring compared to the potential at $300 \mathrm{~K}$ (Figure 2(b)). All sub-lattices including the PrPr dumbbells with a separation of only $0.59 \AA$, pure oxygens and O-Sc-O triple atoms are resolved. The 
diffractogram of the phase image shows an isotropic information transfer up to $4.39 \AA^{-1}$, corresponding to 23 $\mathrm{pm}$ in real space. Quantitative analysis of the atomic column width reveals that the blurring from the instrument is smaller than $20 \mathrm{pm}$, which is smaller than the intrinsic broadening from thermal fluctuations. The method also allows for direct measurements of local Debye-Waller factors of atoms near defects or interfaces [11].
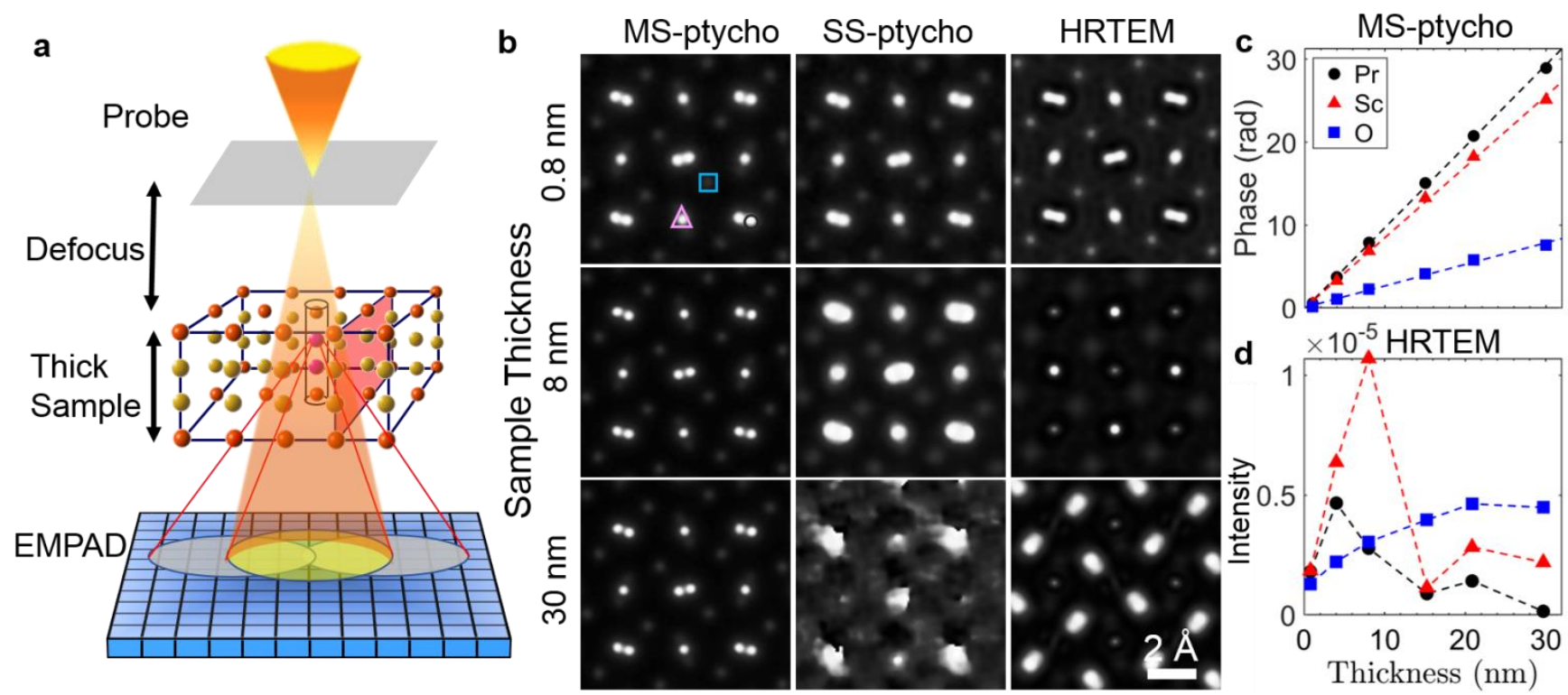

Figure 1. Linear phase-contrast imaging using multi-slice electron ptychography. (a) Setup of 4D-STEM experiments using a defocused probe illumination. (b). Thickness dependent contrast from different phasecontrast imaging methods via simulations: Multislice ptychography (MS-ptycho), single-slice ptychography (SS-ptycho), and conventional high-resolution TEM (HRTEM) with an information limit of $1.25 \AA-1$. (c). Thickness evolution of the intensity at atomic columns from mult-islice ptychography and HRTEM.

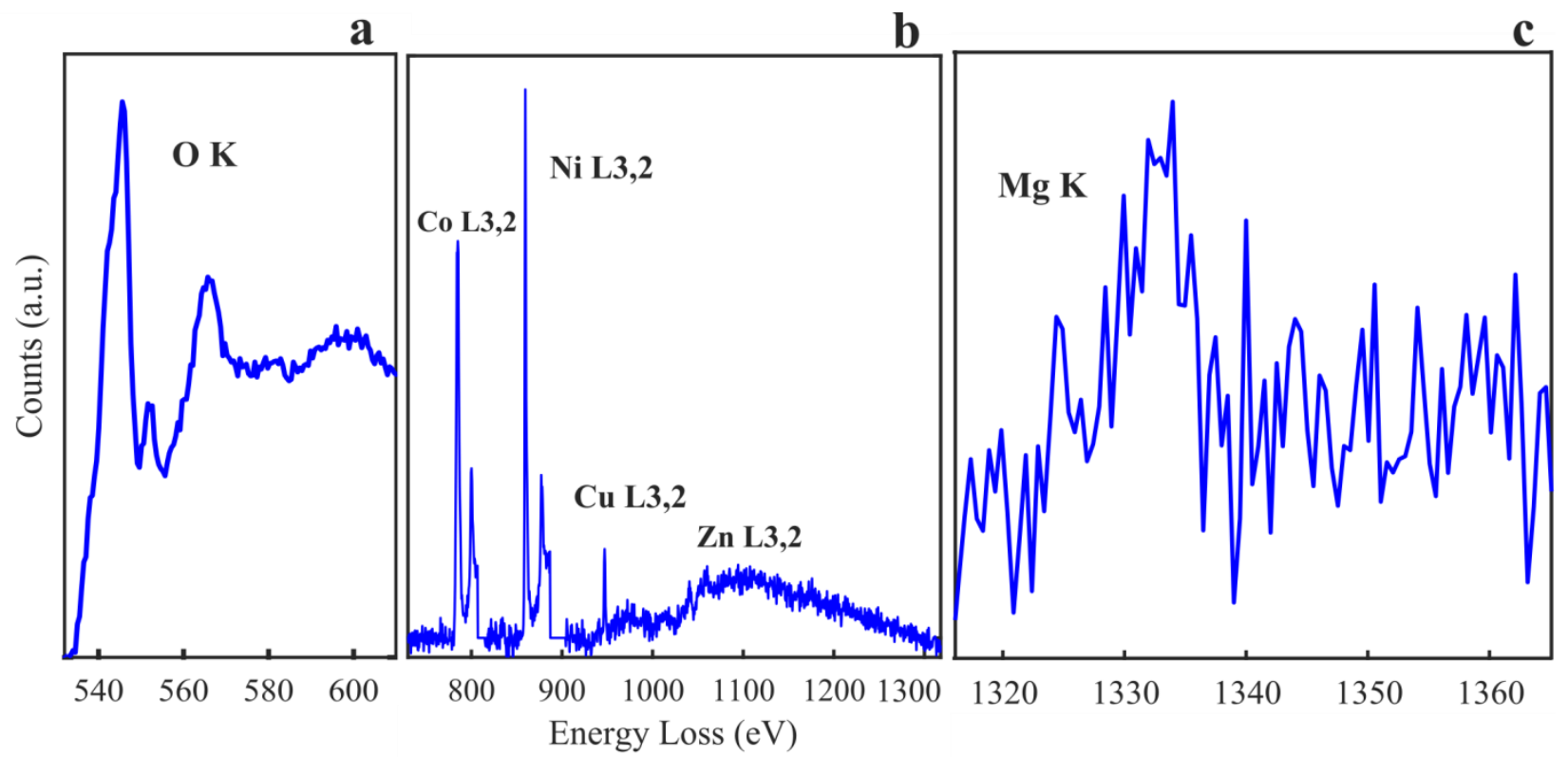

Figure 2. Lattice-vibration-limited resolution from multislice ptychography. (a). Multislice ptychographicallyreconstructed phase image from an experimental dataset acquired from a $21 \mathrm{~nm}$ thick PrScO3 sample. (b)-(d). Simulated projected potential of PrScO3: (a) static potential neglecting zero-point fluctuations, (b) at $300 \mathrm{~K}$, and (c) reconstructed from multislice ptychography using simulated diffraction data at $300 \mathrm{~K}$ including thermal 
fluctuations for $21 \mathrm{~nm}$ thick PrScO3. (e) and (f). Diffractogram of the phase image from experiment: (e) 2D and (f) a line profile along the white dashed line on (e). Phase images in (a) and (d) are normalized to a unitcell thickness of $8.03 \AA$.

\section{References}

[1] MA O'Keefe, Ultramicroscopy 108 (2008), p. 196-209.

[2] R Erni, et al., Physical Review Letters 102 (2009), p. 096101.

[3] H Sawada, et al., Journal of Electron Microscopy 58 (2009), p. 357-361.

[4] R Hovden, et al., Physical Review B, 86 (2012), p. 195415.

[5] Y Jiang, et al., Nature 559 (2018), p. 343.

[6] S Gao, et al., Nature Communications 8 (2017), p. 163.

[7] Y Jiang, et al., Microscopy and Microanalysis 24 (2018), p. 192-193.

[8] M Schloz, et al., Optics Express 28 (2020), p. 28306-28323.

[9] HG Brown, et al., Physical Review Letters 121 (2018), p. 266102.

[10] Z Chen, et al., arXiv: 2101.00465 (2021).

[11] Research supported by US NSF (grants DMR-1539918 and DMR-1719875). 